\title{
Why a Catheter Can Be Correctly Placed in the Anterior Horn of Lateral Ventricle by Inserting Perpendicular to the Frontal Bone on the Ventricular Drainage? Demonstration of the Accuracy of an Inserting Path by Computed Tomographic Image Study and Clinical Practices
}

\author{
Kiyonobu IKEDA, ${ }^{1}$ Takashi ASAHI, ${ }^{1}$ Takaaki IIDA, ${ }^{1}$ Jiro YAMAmoto, ${ }^{1}$ \\ Tsuyoshi TsUKADA, ${ }^{1}$ Nobutaka YAMAMOTO, ${ }^{1}$ Fumihiko TAKEUCHI, ${ }^{1}$ \\ Shigeru Munemoto, ${ }^{1}$ Shu-ji SATO, ${ }^{1}$ Shuichi AKaIKe, ${ }^{2}$ and Katsuo SHOIN ${ }^{2}$ \\ ${ }^{1}$ Department of Neurosurgery, Kanazawa Neurosurgical Hospital, \\ Nonoichi, Ishikawa, Japan; \\ ${ }^{2}$ Department of Neurosurgery, Kanazawa Medical Center, \\ Kanazawa, Ishikawa, Japan
}

\begin{abstract}
Why a catheter can be correctly placed in the ventricle by inserting perpendicular to the frontal bone on the ventricular drainage? We performed a study on the accuracy of a path perpendicular to the skull surface into the anterior horn using computed tomography (CT), and a clinical study. Twenty patients were studied on CT images. Using the curved multi-planar reconstruction method, the curved frontal skull and brain were reconstructed to flat structures, and perpendicular lines were drawn from the flat surface to the foramen of Monro on the reconstructed images. In clinical practice, we made a device which guided a catheter inserting perpendicular to the frontal skull surface, and used it in the ventricular drainage surgery for 148 hydrocephalic patients (158 surgeries). We discovered that the curved surface of the frontal bone around Kocher's point represents the surface of a globe (mean radius, $75.9 \pm 4.3 \mathrm{~mm}$ ) centering on the foramen of Monro. The distribution of points ranged from 13.5-43.5 mm (mean, $43.5 \pm 6.1 \mathrm{~mm}$ ) to the midline, with points appearing more laterally as ventricular size increased. A catheter was placed in the ventricle in 148 surgeries $(99.4 \%)$, and the catheter reached the ventricle with correct orientation toward the foramen of Monro in $128(81.0 \%)$. The reason why the ventricular insertion perpendicular to the frontal bone surface can provide a consistent path toward the foramen of Monro is that the curved surface of the frontal bone around Kocher's point represents the surface of a globe centered on the foramen of Monro.
\end{abstract}

Key words: ventricular tap, guide, frontal bone

\section{Introduction}

Ventricular catheter placement has usually been performed through a burr hole created at Kocher's point and according to the landmarks of the medial canthus of the ipsilateral eye in the frontal plane and a point just in front of the external auditory meatus in the lateral plane. ${ }^{1,2)}$ Tips for these surgical procedures involved the following: 1) making the burr hole at Kocher's point; and 2) inserting the catheter perpendicular to the frontal bone.

Received June 15, 2016; Accepted November 18, 2016
However, even these simple procedures sometimes become difficult after dressing over or rotating the patient's head. Aiming to allow easy performance of ventricular tap without the need for landmarks, Ghajar et al. ${ }^{3)}$ developed a device for ventricular catheter placement, which guides a catheter in a path perpendicular to the skull surface and to enter the ventricle in the midpupillary line. Although these procedures has been performed on the basis of clinical successful experiences, why a catheter can be correctly placed in the anterior horn of lateral ventricle by inserting perpendicular to the frontal bone at Kocher's point on the ventricular 
drainage? In order to seek a theoretical answer to this question, we studied on the accuracy of a path perpendicular to the skull surface from Kocher's point towards the anterior horn using images from computed tomography (CT) in patients with hydrocephalus. And, in order to demonstrate the accuracy, we made a device for easy placement of a ventricular catheter like Ghajar's one ${ }^{3,4)}$ and applied it in our clinical practices.

\section{Materials and Methods}

\section{CT image study}

For twenty patients (10 patients with dilated ventricle, 10 with normal ventricle; age range, 12-85 years), Evans index (anterior horn width / cranial width), various points near Kocher's point on the frontal bone from which perpendicular lines were drawn toward the foramen of Monro, and distance from those points on the frontal bone to the foramen of Monro were studied on CT images. Using the curved multi-planar reconstruction (CPR) method (Software: “AZE Virtual Place”), plotting points on the curved skull surface on the CT images are interpolated automatically to smooth flat shape (CPR image) (Fig. 1A), and cross section line is displayed on the CPR image on the work station. Those plotting points on the frontal bone at which perpendicular lines passed through the anterior horn and reached the foramen of Monro were studied.

\section{A guiding device for anterior ventricular tap}

We made a guiding device for anterior ventricular tap (Fig. 2) like Ghajar's one, ${ }^{3)}$ which guided a catheter inserting perpendicular to the skull surface at Kocher's point. Our device is rigid and measures 3.5 $\mathrm{cm}$ in height, and the bottom part is $1.7 \mathrm{~cm}$ in outer diameter and $3.2 \mathrm{~mm}$ in inner diameter, designed for using the ventricular tap needle used in our institutes (length, $10 \mathrm{~cm}$; outer diameter, $3.0 \mathrm{~mm}$ ). The bottom part of the device is wide with a ring-shaped outline to facilitate a perpendicular entry into the bone surface.
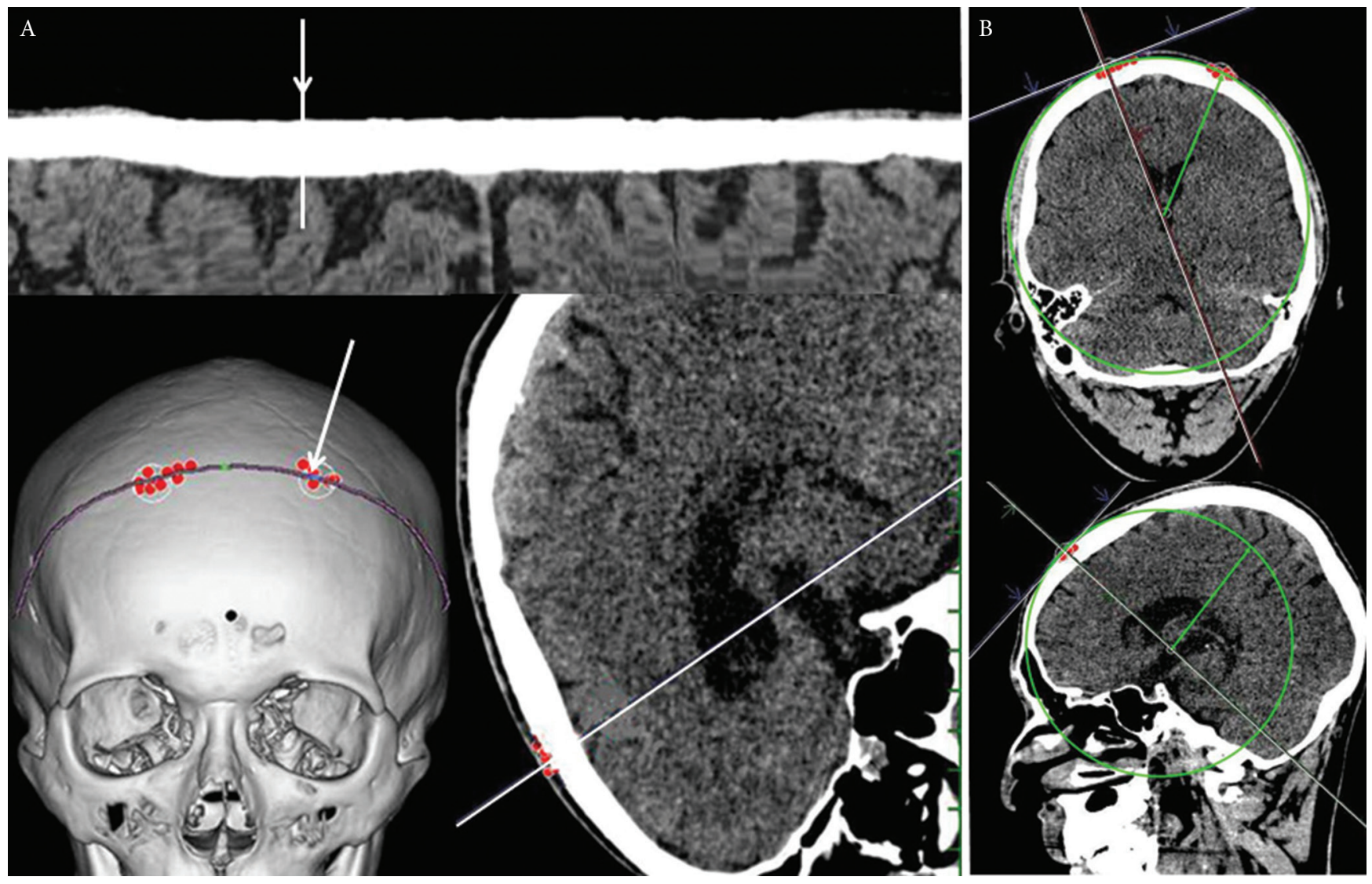

Fig. 1 CT image study. (A) Using the curved multi-planar reconstruction (CPR) method, curved frontal skull and brain on CT images were reconstructed to flat structures. Lines perpendicular to the flat surface were drawn, and starting points from which lines passed through the anterior horn and reached the foramen of Monro were plotted on the frontal bone. The distribution of these points and distance from these points to the foramen of Monro were studied. (B) The curved surface of the frontal bone at those points was nearly identical to the surface of a globe centered on the ipsilateral foramen of Monro. The globe had a radius of $75.9 \pm 4.3 \mathrm{~mm}$. 


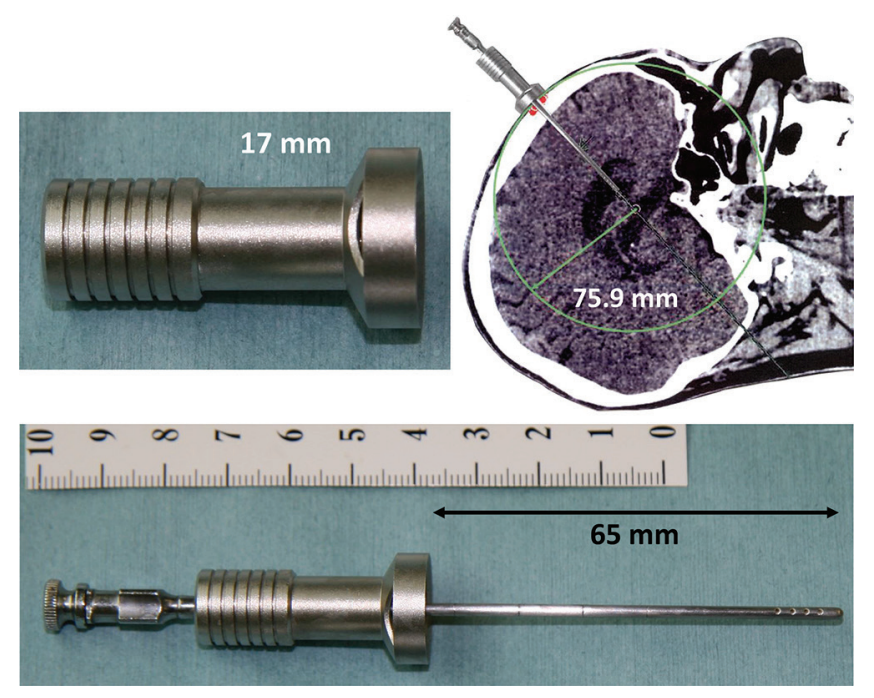

Fig. 2 Our guiding device for ventricular tap.

\section{Clinical practice}

The clinical study using a guiding device was approved by our hospital ethics committee. For the 9 years from January 2007 to October 2015, a total of 148 patients with a mean age of $66.2 \pm 15.3$ years (range, 14-85 years) underwent 158 ventricular tapping surgeries using our guiding device. These involved acute hydrocephalus following subarachnoid hemorrhage in 58 cases, intraventricular hemorrhage in 41 , obstructive hydrocephalus by posterior fossa lesions in 24 , chronic hydrocephalus in 22 , and cerebral infarction (decompressive ventricular drainage) in 3.

After the frontal bone is perforated at Kocher's point (which is marked before draping on the frontal scalp at $2.5 \mathrm{~cm}$ from the midline, approximately $11 \mathrm{~cm}$ posterior to the nasion, and at least $1 \mathrm{~cm}$ anterior to the coronal suture on the frontal bone) in all cases and the dura was opened, the guiding device was set on the burr hole (approximately 1.2 $\mathrm{cm}$ in diameter). Through the device, a ventricular tap needle was inserted perpendicular to the skull surface into the brain, $6.5 \mathrm{~cm}$ deep to the skull surface. After confirmation of cerebrospinal fluid coming up and keeping the needle inserted for a few ten seconds, both the tap needle and guiding device were removed, and a ventricular catheter was inserted $4.0-6.0 \mathrm{~cm}$ from the surface of the brain cortex through the path made by needle. After surgery, postoperative CT was always performed.

\section{Results}

\section{CT image study}

Possible points for insertion of a ventricular tap toward the foramen of Monro were shown on the frontal skull surface (Fig. 1A). The distribution of these points ranged from $13.5 \pm 2.5$ to $43.5 \pm 6.1$ $\mathrm{mm}$ from the midline, with the most lateral points appearing more laterally as ventricle size increased (Fig. 3). The most lateral points were correlated to Evans index $(\mathrm{B}=11.528,1.016$, simple regression analysis, $P=0.02$ ) (Fig. 4). The finding that many lines perpendicular to the frontal bone reached the foramen of Monro showed that the curved surface of the frontal bone including these points resembled the surface of a globe centering on the ipsilateral foramen of Monro. This globe had a mean radius of $75.9 \pm 4.3 \mathrm{~mm}(\mathrm{n}=20$; Fig. $1 \mathrm{~B})$.

\section{Clinical practice of our guiding device}

Our device is $3.5 \mathrm{~cm}$ in height, and ventricular tap needle $10 \mathrm{~cm}$ long is used. The frontal bone is approximately $1.0 \mathrm{~cm}$ thick, and the needle part protrudes $6.5 \mathrm{~cm}$ from the device. The needle should be inserted perpendicular to the brain surface and $5.5 \mathrm{~cm}$ deep into the brain. A catheter was placed in the ventricle in 148 surgeries (99.4\%), and the catheter reached the ventricle with correct orientation toward the foramen of Monro in 128 $(81.0 \%)$ (Fig. 5). In the other 30 surgeries, the catheter was not correctly oriented toward the foramen of Monro, caused by a shift of the catheter tip compressed by intraventricular hematoma in 8, ventricular shift compressed by intracerebral hematoma in 5 , misinsertion of the catheter not along the correct path made by the ventricular needle in 2, inappropriate location of the burr hole in 8 , and skull deformity in 7 (oxycephaly-like deformity of symmetrical frontal bone surface with radius curvature of 59.0 and $62.4 \mathrm{~mm}$, respectively, in 2; brachycephaly-like deformity with radius curvature of 91.4 and $98.2 \mathrm{~mm}$, respectively, in 2 ; and asymmetrical frontal bone deformity with different radius curvature in 3) (Fig. 6). In these seven cases with skull deformity, frontal bone surfaces were not thought to adequately resemble a globe centering on the foramen of Monro.

\section{Discussion}

Usually, a burr hole for ventricular tap is made at Kocher's point just anterior to the coronal suture (1 $\mathrm{cm}$ anterior to the coronal suture or $10-12 \mathrm{~cm}$ from the glabella) and $2.0-3.0 \mathrm{~cm}$ from the midline. ${ }^{1)}$ Landmarks for catheter insertion are the medial canthus of the ipsilateral eye in the frontal plane and a point just in front of the external auditory meatus in the lateral plane. ${ }^{2)}$ Manual surgical procedures for ventricular tap usually performed by most neurosurgeons are very simple, but a ventricular needle tip does not always reach the lateral ventricle. 

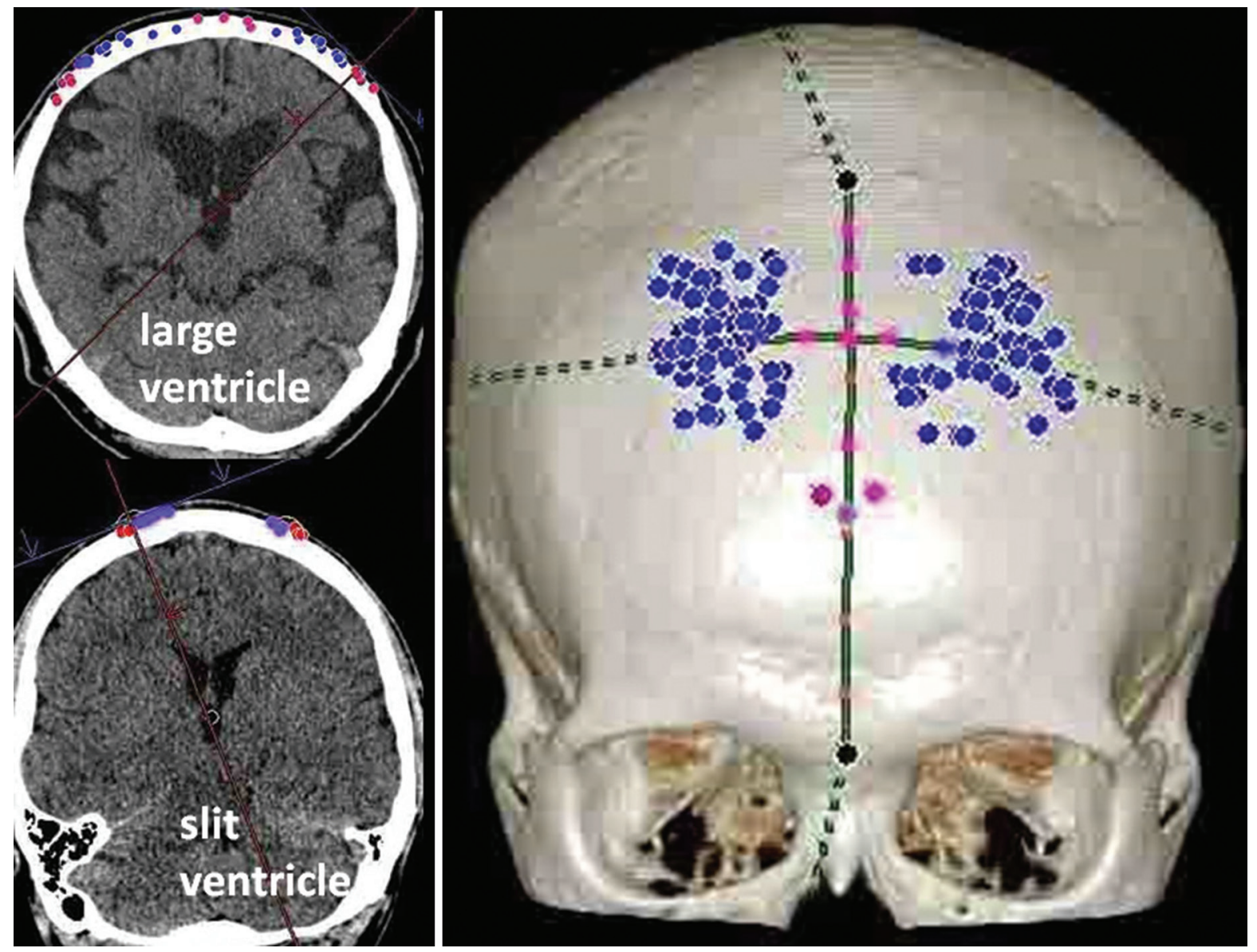

Fig. 3 A distribution of possible points for ventricular tap oriented toward the foramen of Monro (shown as blue dots). The distribution ranges from $13.5 \mathrm{~mm}$ to $43.5 \mathrm{~mm}$ from the midline, with the most lateral points appearing more lateral as ventricular size increased.

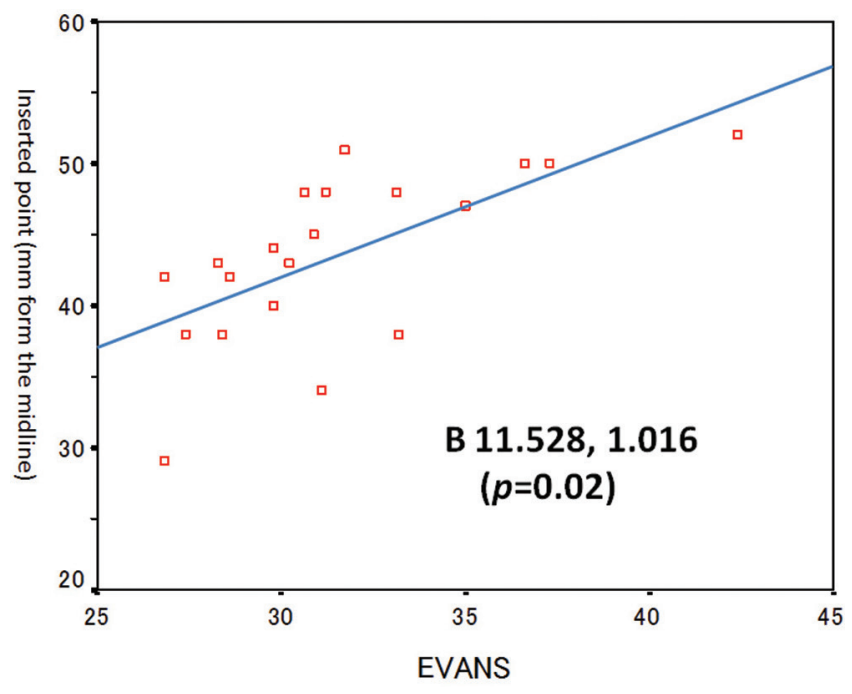

Fig. 4 Relationship between the most lateral suitable points and Evans index. Suitable points correlated with Evans index $(B=11.528,1.016$, simple regression analysis, $P=0.02$ ).
Such surgical failures are typically caused by the following: 1) difficulties in identifying positional relationships between the landmarks of the external meatus, nasion, and tip of the nose for needle insertion on the drape over the patient's head; 2) size of the ventricle (slit or small ventricle); and 3) congenital or acquired cranial deformities after craniotomy.

As a rapid bedside technique for ventricular catheter placement in children without the help of the standard landmarks, McWilliam et al. ${ }^{5)}$ developed a drill hole approach, through which a bone marrow needle was used for perforation of the skin, frontal bone, and dura perpendicular to their surfaces. Ghajar $^{3)}$ designed a guiding device for ventricular catheter placement along a path perpendicular to the bone surface. The body of the lateral ventricle lies in the midpapillary line and the curve of the superior aspect of the anterior horn parallels the curve of the overlying cranium. Insertion of a ventricular tap needle perpendicular to the frontal bone at 

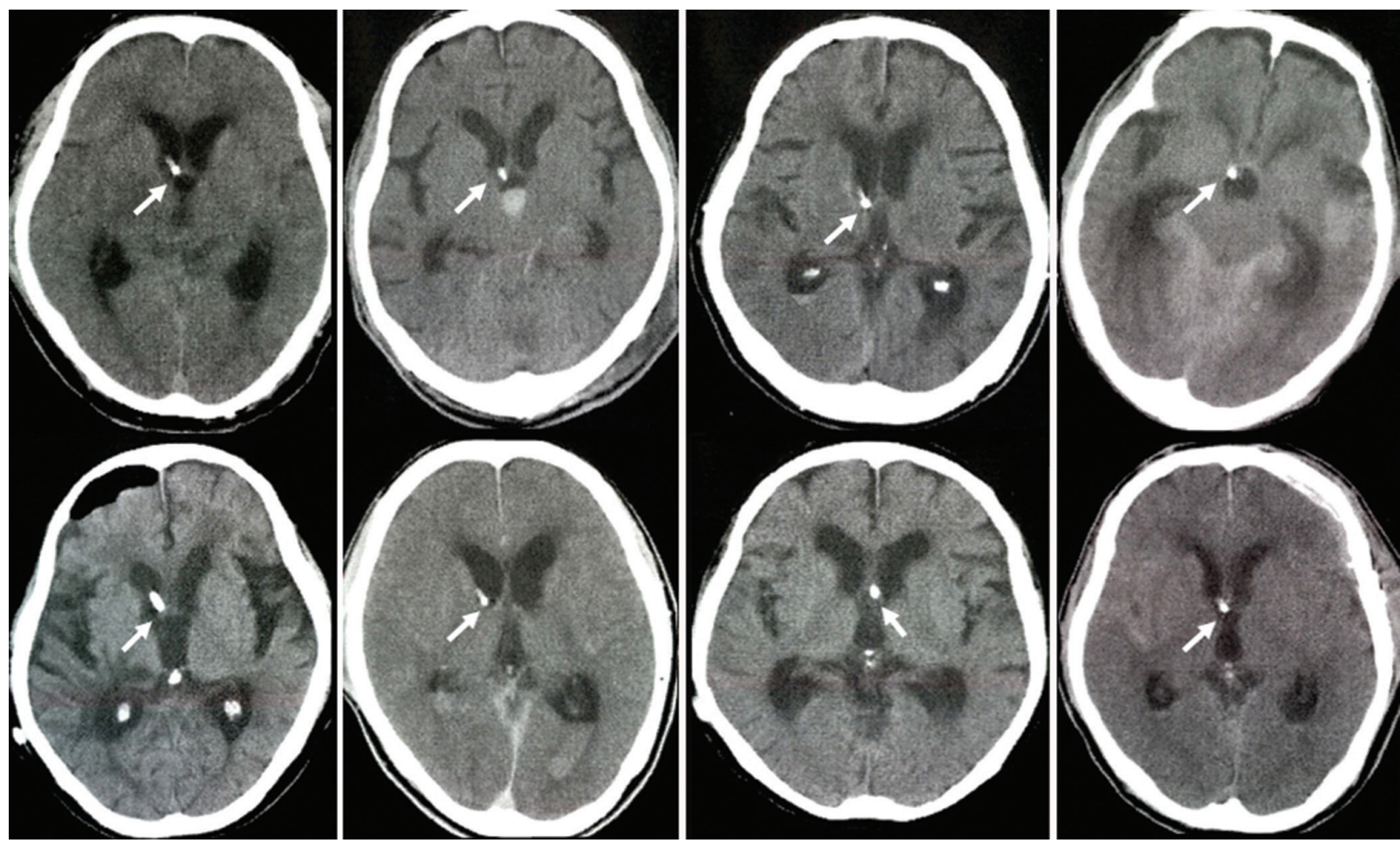

Fig. 5 Representative cases of successful placement of a ventricular catheter oriented correctly toward the foramen of Monro. Arrows show the tips of the catheters.

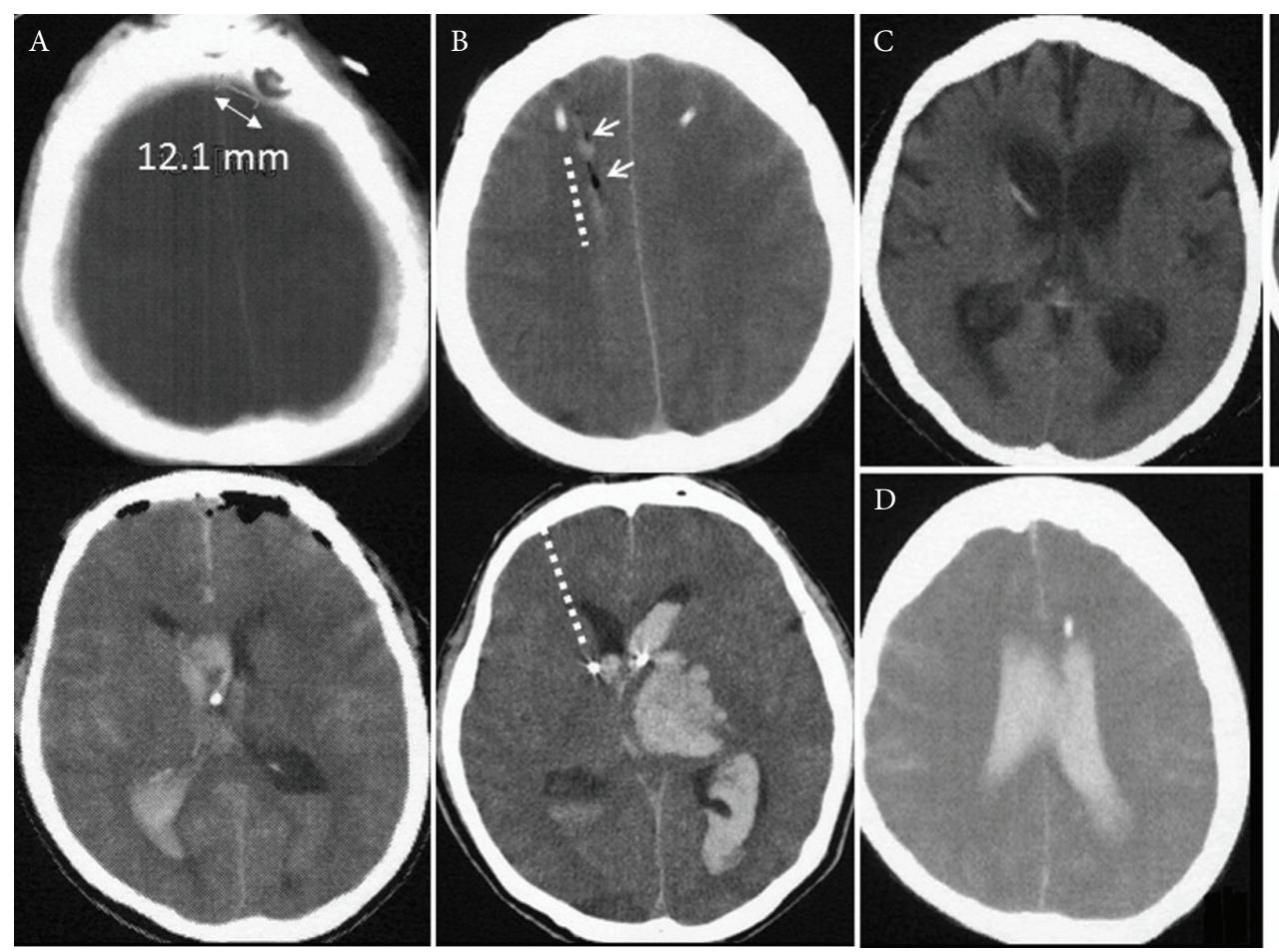

Fig. 6 Unsuccessful cases of ventricular catheter placement missing the foramen of Monro. Causes of incorrect placement were as follows: (A) inappropriate location of the burr hole (very near burr hole $12.1 \mathrm{~mm}$ from the midline); (B) insertion of a catheter (dotted lines) not along the correct path made by the ventricular needle (small arrows); and (C-E) cranial deformities (C: brachiocephalic deformity; D: oxycephalic deformity; E: asymmetrical cranial deformity). 
Kocher's point independent of the landmarks can thus make a path perpendicular to the upper wall of the anterior horn of the lateral ventricle. Our CT image study found why a path perpendicular to the frontal bone points toward the foramen of Monro, and revealed that the curved surface of the frontal bone near Kocher's point resembles the surface of a globe measuring $75.9 \mathrm{~mm}$ in radius with the foramen of Monro at the center. Concerning the accuracy of the perpendicular line angle in the CPR method, that is hardly affected by skewness, especially on the curve with high circle degree like a frontal bone, and therefore it is thought that the use of the CPR method in this study is proper.

In our clinical practices, catheters were placed in the anterior horn in $99.4 \%$ of surgeries and were correctly oriented toward or reached the foramen of Monro in $81.0 \%$. From the results of 30 cases in which a catheter was not correctly oriented toward the foramen of Monro, it is thought that a ventricular tap needle should be inserted at the Kocher's point (20-30 mm to the midline) in principle for all cases (with radius curvature of $75.9 \pm$ $4.3 \mathrm{~mm}$ ) with ventricular dilatation. However, for cases with cranial deformities, insertion point should be corrected around Kocher's point; mainly $30 \mathrm{~mm}$ to the midline for cases with the oxycephaly-like frontal bone with smaller radius (around $60 \mathrm{~mm}$ ), mainly $20 \mathrm{~mm}$ for cases with the brachycephalylike frontal bone with larger radius (around 95 $\mathrm{mm}$ ), and insertion at the side of frontal bone with radius around $75.9 \mathrm{~mm}$ for cases with asymmetrical cranial deformity. These clinical results show that neurosurgeons can easily perform ventricular tap using the guiding device even when the head is draped or rotated, and when ventricular drainage is unexpectedly needed for brain decompression. As further clinical applications, the correct orientation toward the foramen of Monro by this guiding device instead of a navigation system might permit easy creation of a path for endoscopic surgery for intrathird ventricular lesions and third ventriculostomy through the foramen of Monro.

Our CT image study showed that possible burrhole points for ventricular tap toward the foramen of Monro range over a distribution from $13.5 \pm$ 2.5 to $43.5 \pm 6.1 \mathrm{~mm}$ from the midline, including the well-known Kocher's point. Yosikai et al.4) clinically experienced successful ventricular tap pointing toward the foramen of Monro from the coronal suture to just $4 \mathrm{~cm}$ anterior to the suture and $2.5-4.5 \mathrm{~cm}$ from the midline. In cases with a larger ventricle, the possible burr-hole points are more lateral to the midline, and in cases with a slimmer ventricle, the points are nearer the midline. In cases in which ventricular drainage is preoperatively expected for brain decompression, the bregma and midline should be marked before draping, at the very least.

The device for ventricular tap, which guides a catheter inserting perpendicular to the frontal bone surface of a globe centered on the foramen of Monro, can help neurosurgeons in the insertion of a ventricular tap needle toward the foramen of Monro and can release us from surgical difficulties achieving ventricular tap in complicated cases.

\section{Conclusion}

We performed a CT image study and clinical practice of the guiding device aiming to seek the reason why the ventricular insertion perpendicular to the frontal bone surface can provide a consistent path toward the foramen of Monro. We discovered that the curved surface of the frontal bone around Kocher's point represents the surface of a globe centered on the foramen of Monro.

\section{Conflicts of Interest Disclosure}

The authors have no personal, financial, or institutional interest in any of the materials or devices used in the article. All authors who are members of The Japan Neurosurgical Society (JNS) have registered online Self-reported COI Disclosure Statement Forms through the website for JNS members.

\section{References}

1) Friedman WA, Vries JK: Percutaneous tunnel ventriculostomy. Summary of 100 procedures. J Neurosurg 53: 662-665, 1980

2) Ojemann RG, Black PM: Hydrocephalus in adults, in Youmans JR (ed): Neurological Surgery, ed 2. Philadelphia, WB Sunders, 1982: 1423-1435

3) Ghajar JB: A guide for ventricular catheter placement. Technical note. J Neurosurg 63: 985-986, 1985

4) Yoshikai S, Hashiguti K, Harata N: A design for an inexpensive ventricular tap device utilizing the frontal region approach. Japanese Congress of Neurological Surgeons 12: 196-198, 2003 (Japanese)

5) McWilliam RC, Stephenson JB: Rapid bedside technique for intracranial pressure monitoring. Lancet 2: 73-75, 1984

Address reprint requests to: Kiyonobu Ikeda, MD, PhD, Department of Neurosurgery, Kanazawa Neurosurgical Hospital, 262-2 Goh-machi, Nonoichi, Ishikawa 921-8841 Japan.

e-mail: kn-ikeda@med.email.ne.jp 